\title{
Towards ASTEP+, a two-color photometric telescope at Dome C, Antarctica
}

Nicolas Crouzet ${ }^{\mathrm{a}}$, Abdelkrim Agabi ${ }^{\mathrm{b}}$, Tristan Guillot ${ }^{\mathrm{b}}$, Lyu Abe ${ }^{\mathrm{b}}$, François-Xavier Schmider ${ }^{\mathrm{b}}$, Djamel Mékarnia ${ }^{\mathrm{b}}$, Amaury H.M.J. Triaud ${ }^{\mathrm{c}}$, Yves Bresson ${ }^{\mathrm{b}}$, Nicolas Mauclert ${ }^{\mathrm{d}}$, Christophe Bailet $^{\mathrm{b}}$, Dennis Breeveld ${ }^{\mathrm{a}}$, Sander Blommaert ${ }^{\mathrm{a}}$, Brian Shortt ${ }^{\mathrm{a}}$, Jean-Baptiste Daban ${ }^{\mathrm{e}}$, Anne-Marie Lagrange ${ }^{f}$, Romain Touzé ${ }^{g}$, Justin Dufour ${ }^{g}$, Valentin Stee $^{g}$, and Jocelyn Caruana ${ }^{g}$

${ }^{a}$ European Space Agency (ESA), European Space Research and Technology Centre (ESTEC), Keplerlaan 1, 2201 AZ Noordwijk, The Netherlands

bUniversité Côte d'Azur, Observatoire de la Côte d'Azur, CNRS, Laboratoire Lagrange, CS 34229, F-06304 Nice Cedex 4, France

${ }^{\mathrm{c}}$ School of Physics \& Astronomy, University of Birmingham, Edgbaston, Birmingham, B15 2TT, United Kingdom

${ }^{d}$ Observatoire de la Côte d'Azur, Boulevard de l'Observatoire CS 34229, F-06304 Nice

Cedex 4, France

'Observatoire Midi-Pyrénées, 14 avenue Edouard Belin, 31400 Toulouse, France

${ }^{\mathrm{f}}$ LESIA, Observatoire de Paris, Université PSL, CNRS, Sorbonne Université, Université de

Paris, 5 Place Jules Janssen, 92195 Meudon, France

gSophia Engineering, 5 rue Soutrane, 06560 Sophia Antipolis, France

\begin{abstract}
Dome C, Antarctica is unique in particular for long-duration astronomical observations due to the excellent weather conditions and nearly uninterrupted nights during the Southern winter period. The $40 \mathrm{~cm}$ telescope ASTEP has been operating successfully at the Concordia base, at Dome C, since 2010. We describe the new ASTEP+, a major upgrade of its camera box which will allow it to observe simultaneously in two colors. Approximately three times more photons will be collected for science, yielding more sensitive and accurate observations. The southern location of the telescope means that it is ideally located to follow-up exoplanetary targets in preparation for the future JWST and Ariel observations, in particular when located in the southern continuous viewing zones of these space-based telescopes.
\end{abstract}

Keywords: Photometry, Times-series, Exoplanets, Transits, Antarctica, ASTEP

\section{INTRODUCTION}

Dome C in Antarctica offers unique conditions on Earth for astronomical observations. The 3-month continuous night during the Antarctic winter enables uninterrupted observations. The atmospheric conditions are excellent in particular during the winter. Thanks to its location on a summit of the high Antarctic plateau at an altitude of $3233 \mathrm{~m}, 1100 \mathrm{~km}$ away from the coast, this site is not affected by katabatic winds. The air is extremely dry and cold, the pressure is low (the equivalent altitude is around $4000 \mathrm{~m}$ ), so the atmospheric transmission is increased in the visible and even more in the infrared compared to other sites on Earth. ${ }^{1,2}$ The wind speed is low (generally a few $\mathrm{m} / \mathrm{s}$ ), the sky is photometric $67 \%$ of the time during the observing season, which is comparable to La Silla and Mauna Kea, and scintillation is decreased. ${ }^{3-6}$ These conditions are highly beneficial to time-series observations. Dome $\mathrm{C}$ is the location of the French-Italian Concordia station which is inhabited all year long since 2005. The ASTEP project (Antarctic Search for Transiting ExoPlanets, ${ }^{7}$ PI: T. Guillot,

Further author information: (Send correspondence to Nicolas Crouzet or Abdelkrim Agabi)

Nicolas Crouzet: E-mail: nicolas.crouzet@esa.int, Telephone: +31 715655132

Abdelkrim Agabi: E-mail: agabi@unice.fr, Telephone: +33 (0)4 92076327 
Laboratoire Lagrange, CNRS, Nice, France) aims at detecting and characterizing transiting exoplanets from this site. A $40 \mathrm{~cm}$ Newton telescope was designed and built to perform high precision photometry under the extreme conditions of the Antarctic winter. ${ }^{8}$ The ASTEP telescope is equipped with a front-illuminated $4096 \times$ 4096 pixel CCD camera (FLI Proline KAF 16801E) for science observations and a SBIG camera for guiding; a dichroic filter sends the red wavelengths to the science channel and the blue wavelengths to the guiding channel. This telescope was installed at Concordia in 2010 and provided excellent data since then. ${ }^{9-13}$ Here, we present the ongoing upgrade of the system, which consists in two new cameras and a new camera box for simultaneous observations at visible and red to near-infrared wavelengths.

\section{SCIENCE GOALS}

The broad objective of ASTEP is to discover and characterize transiting exoplanets. We will conduct observations following four main axes of research.

a) Terrestrial exoplanets. One axis is the detection of transiting terrestrial exoplanets, in particular around red dwarfs. A hot topic in the exoplanet field is the detection of terrestrial exoplanets with atmospheres that can be characterised. This requires small and relatively bright host stars. M dwarfs are the most favorable targets to search for terrestrial exoplanets: their small size and low mass yield deeper planetary transits and larger radial velocity amplitudes, the habitable zone is closer to the star which increases the transit probability for planets in this region, they account for $75 \%$ of stars in the Galaxy, and the occurrence rate of small planets around them is high (at least 1.4 Earth-size planets per star ${ }^{14-16}$ ). The potential for characterising their atmospheres via transit spectroscopy is also enhanced. Their emission peak is around $800-900 \mathrm{~nm}$. The new setup of ASTEP will include a red-enhanced, back-illuminated camera specifically chosen to observe M dwarfs (see Section 3).

b) Long period exoplanets. Another goal is the detection of warm and cold giant exoplanets, with orbital periods typically longer than 30 days, which remain a poorly constrained population. Studying this population would put our knowledge of the physics of close-in gas giant planet and of their atmospheres into perspective. Observations from Dome $\mathrm{C}$ are particularly useful for long transits, generally corresponding to long period objects, which cannot be covered fully from temperate sites. An example of such observation is given in Figure 1. We will also search for terrestrial planets with long orbital periods, potentially in the habitable zone of their stars. Again, M dwarfs are the most favorable targets to find such planets.

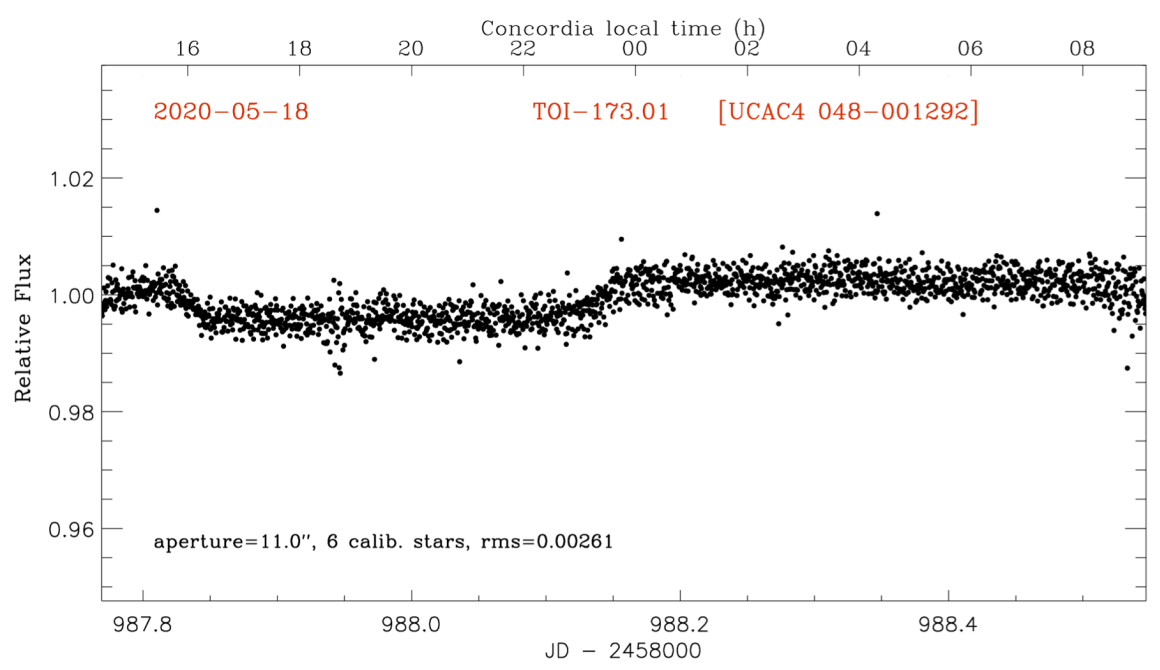

Figure 1. Lightcurve of the 29.75 day period gas-giant exoplanet candidate TOI-173.01 obtained by ASTEP at Concordia on 18th May, 2020. This object orbits a bright, F-type star (Vmag $\left.=9.32, \mathrm{~T}_{\mathrm{eff}} \approx 6680 \mathrm{~K}\right)$ and was discovered by the TESS mission. The transit lasts 8 hours and has a depth of $0.63 \%$ corresponding to a radius of $1.1 R_{\text {Jup }}$ for the transiting object. Dome $\mathrm{C}$ is an ideal location to observe such long transits and the new system will make these observations even more efficient. 
c) Exoplanets around young stars. We will also search for transiting exoplanets around young stars (a few Myr). Observing these systems is key to understand the formation and early evolution of planets. Detecting these planets is challenging because young stars are generally active and the planets may be hidden in a circumstellar disk. The two new cameras will transform the current single color telescope into a simultaneous two-color device. The two-color photometry will allow us to distinguish stellar variability and planetary transits: stellar photometric variations are stronger in the blue than in the red whereas exoplanet transits are achromatic at the level of precision we will reach.

d) Circumbinary planets. Long thought to be impossible to form, circumbinary planets (planets orbiting both stars of a binary) were unambiguously detected by Kepler. ${ }^{17}$ So far only a dozen systems have been identified and much remains to be explored. ${ }^{18}$ The properties of circumbinary planets are important to understand the processes of planet formation and of disc-driven migration. ASTEP will participate in several ways: 1 - by targeting predicted planet transit times of systems identified with TESS, 2 - by attempting to detect the transits of circumbinary planets discovered with radial-velocities, and 3 - by collecting binary eclipses of TESS or radialvelocity systems to measure eclipse-timing variations. Circumbinary planet transit durations regularly exceed a typical non-Antarctic night, and can carry large uncertainties, both of which are dealt efficiently with ASTEP.

e) Targets of opportunity. The unique location of ASTEP means that it can also be used for many purposes, including the observation of variable stars, asteroids, comets, occultations by solar system objects or participate in programs to monitor any transient events. The possibility to have a reliable, automatic observatory at Concordia is an asset for many astrophysical programs.

A significant fraction of potential targets for goals a) to d) comes from the NASA TESS mission. ${ }^{19}$ Nearly 2400 "TESS Objects of Interest" (TOIs) have been delivered so far and intensive follow-up observations are needed. ASTEP is part of the TESS Follow-up Program (TFOP) and we observed more than 100 TOIs during the winter 2020; the new setup will make our observations much more efficient. We will continue this program to confirm the nature of the system and to maintain accurate transit ephemerides, which will be crucial for future observations with space-based facilities, in particular JWST and Ariel. For these two space telescopes, the southern observation zone with maximum visibility fully overlaps with ASTEP's visibility zone.

\section{A NEW TWO-COLORS CAMERA BOX}

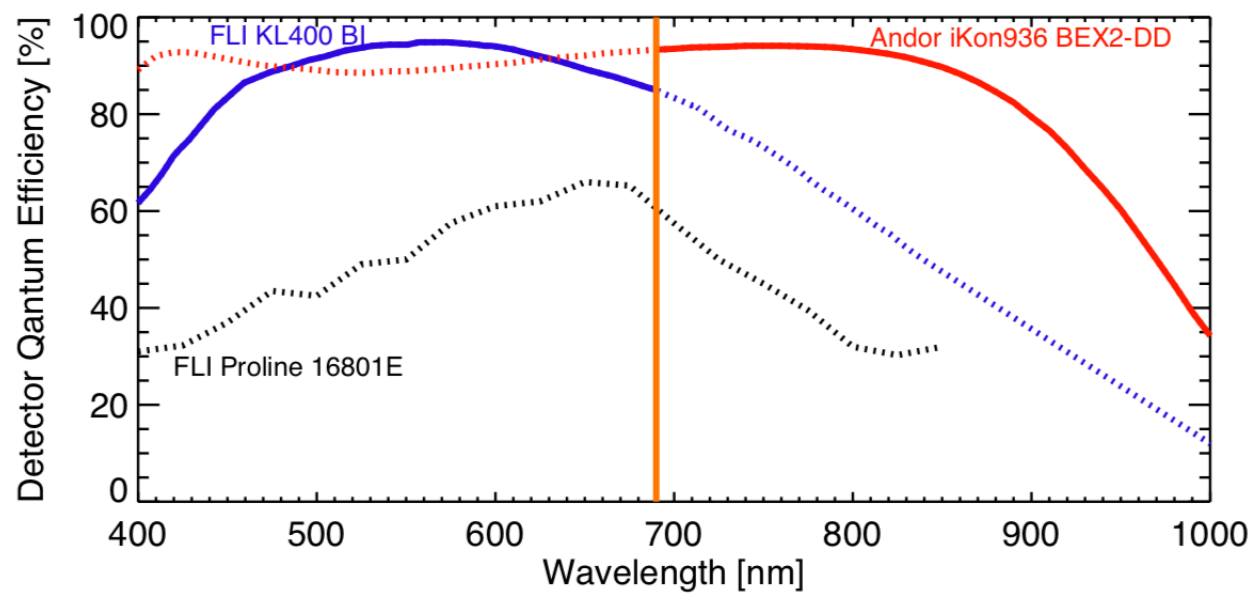

Figure 2. Quantum efficiency of the Andor iKon camera (red) and of the FLI KL 400 camera (blue). A dichroic filter will split the flux at $690 \mathrm{~nm}$. The quantum efficiency of the FLI Proline KAF 16801E camera currently installed on the telescope is shown for comparison (black).

The first focal box of ASTEP included a FLI PL16801E camera at the focal plane, allowing a $1^{\circ} \times 1^{\circ}$ field on 16 Mpxs. The guiding was ensured thanks to a separate small SBIG ME camera imaging the center of the field. 
We decided to change the focal box to allocate two-colors photometry on two separate cameras to increase the bandwidth, get more information on the targets and improve the photometric quality.

The first camera is an iKon-L 936 from Andor (iKon936 BEX2-DD). It has a back-illuminated $2048 \times 2048$ pixel CCD, with deep-depletion, fringe suppression and dual anti-reflection coating, and with a quantum efficiency of $95 \%$ at $800 \mathrm{~nm}$. This high quantum efficiency in the red/near-infrared is ideal to observe cool stars. The same camera is used by the 12 20-cm NGTS telescopes ${ }^{20}$ at Paranal Observatory, and by the six 1-m SPECULOOS telescopes $^{21,22}$ at Paranal, El Teide and San Pedro Mártir Observatories, and which are focused on monitoring ultracool dwarfs. ${ }^{23}$ This camera will be used for science observations. The second camera is a Kepler KL 400 from Finger Lake Instrumentation (FLI). It has a back-illuminated $2048 \times 2048$ pixel Scientific CMOS detector with a quantum efficiency of $95 \%$ at $550 \mathrm{~nm}$. Its high frame rate capability (up to 48 frames per second) and very low reading noise makes it ideal for guiding and its performances are compatible with high quality science imaging. Thus this camera will be used both for guiding and science observations. A dichroic filter will split the incident flux at $690 \mathrm{~nm}$ sending the red/near-infrared wavelengths to the Andor camera and the visible/blue wavelengths to the FLI camera.

With the new cameras, the total flux recorded between 400 and $1100 \mathrm{~nm}$ corresponds to an equivalent bandwidth of $507 \mathrm{~nm}$ (integral of the two QE curves in Figure 2). This has to be compared with the previous ASTEP box, providing an equivalent bandwidth of $150 \mathrm{~nm}$. The transmission of the telescope and the optics (see below) beeing almost unchanged, the photometric gain is about 3.4. This will considerably impact the noise level of our measurements.

\section{OPTICAL DESIGN}

ASTEP's original CCD size, focal scale, the focal ratio and the aperture diameter were defined so as to observation of a large number (thousands) of stars of magnitudes $m_{\mathrm{v}}$ from 8 to 12 while avoiding too much overlap. With its new camera box, ASTEP's main goals remains to discover, monitor and characterize exoplanets. This drives the same technical requirements as previously, ${ }^{8}$ with the addition that accurate photometry now must be possible on both cameras. Two slight modifications with the present setup are to allow for a slightly smaller field of view than the initial $1^{\circ} \times 1^{\circ}$ (at least $0.5^{\circ} \times 0.5^{\circ}$ ) and an in-focus PSF that can have a FWHM that is smaller than one pixel in order to seek higher angular resolution when required.

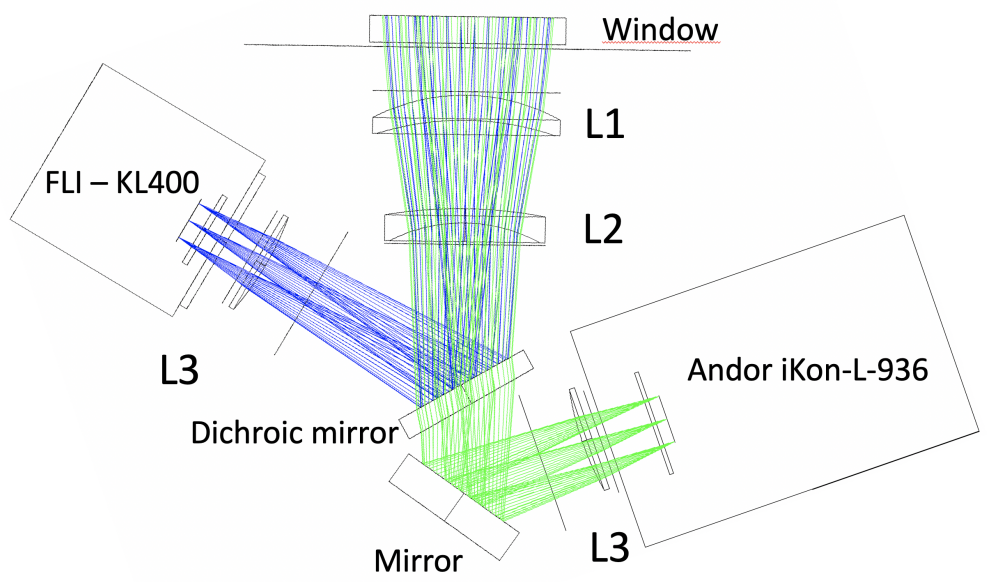

Figure 3. Optical layout.

To ensure a stable and accurate photometry, the optical combination of the Wynne field corrector was optimized under Zemax in order to obtain a PSFs quality as close as possible to the theoretical ones with the same regularity throughout the whole field either in focus or out-of-focus. 
Figure 3 shows the optical layout. A flat entrance window of $110 \mathrm{~mm}$ diameter forms the transition between the cold outer atmosphere and the insulated camera box. A dichroic mirror with a cutting frequency at $690 \mathrm{~nm}$ separates the blue wavelengths, directed towards the FLI camera, and the red wavelengths directed towards a ZERODUR@ flat mirror and the Andor camera.

In order to ensure the correct PSF shapes, a field corrector is formed of a set of three lenses on each optical channel: A $108 \mathrm{~mm}$ convex meniscus lens (L1), a $90 \mathrm{~mm}$ diverging meniscus lens (L2) and a set of two $50 \mathrm{~mm}$ convex lenses for each channel (L3_Andor, L3_FLI). These L3 lenses can be displaced with a translation stage in order to adjust the focal length and obtain the PSF sizes corresponding to the desired scientific program. The field of view obtained on the detectors corresponds to 36' square for the blue channel and 44' square for the red one.

The resulting characteristics of the PSFs in the two channels are shown in Fig. 4, both for the in-focus and out-of-focus modes. An excellent homogeneity of the field with minimal variations as a function of position was obtained over the whole field of the blue channel camera. On the red channel, the optical design leads to a $\sim 10 \%$ variation of the PSF size accross the CCD, which meets specifications. These properties are obtained both in the in-focus and out-of-focus modes. The spot images are regular and there are no optical aberrations.

\section{Red Channel}
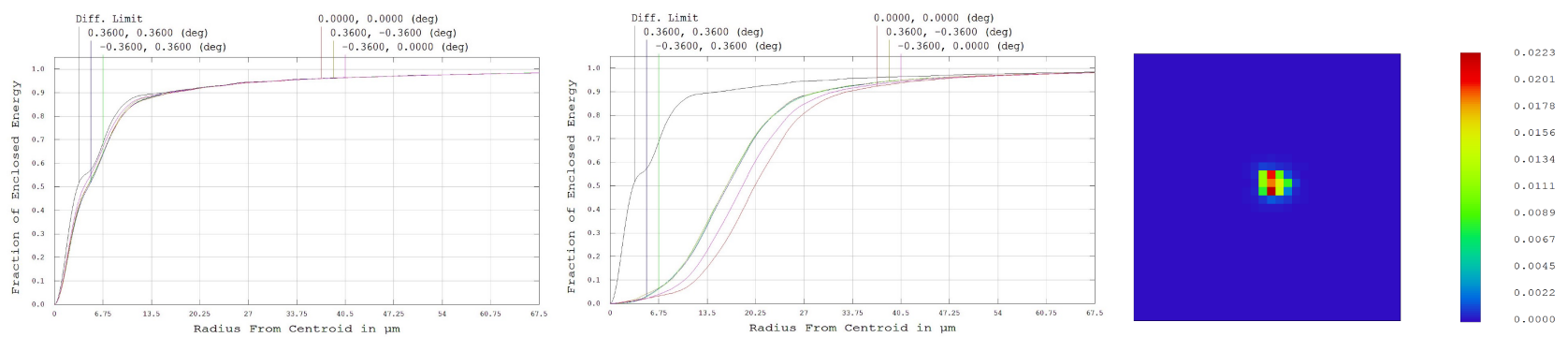

Blue Channel
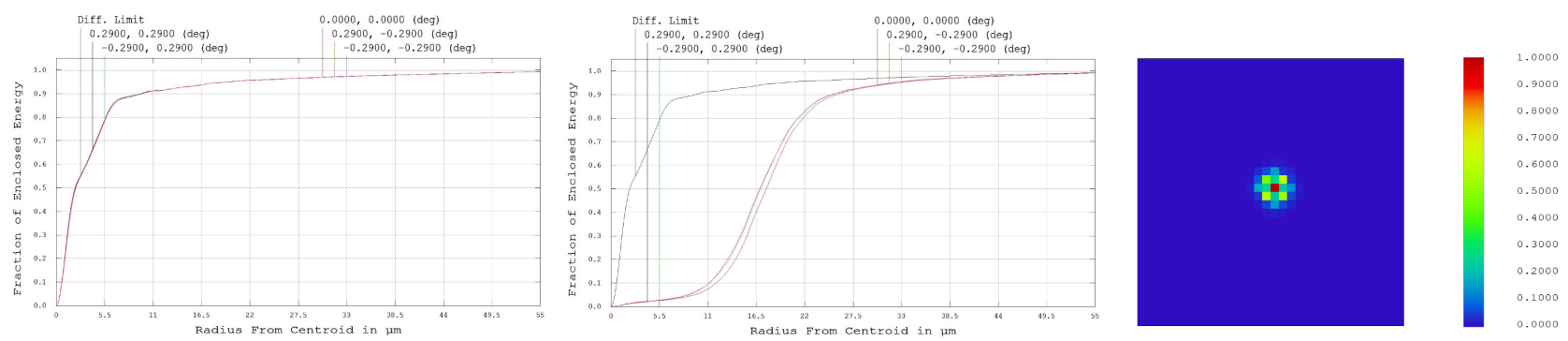

Figure 4. Point spread functions obtained for the red channel (top row) and the blue channel (lower row), when the image is in focus (left column) or slightly out of focus, with a displacement of the last lens by 1 mm relative to its in-focus position (middle and right columns). The left-hand side and middle-column diagrams show the fraction of the energy that is enclosed within a circle of a given radius, in microns. Five sets of lines (often super-imposed) correspond to the images obtained at the center and four corners of the CCDs. The spots on the right column correspond to the energy distributions on the two cameras in units of their pixel sizes. The pixel size is equal to $13.5 \mu \mathrm{m}$ for the red channel camera, and to $11 \mu \mathrm{m}$ for the blue channel camera.

Table 1 provides a quantitative comparison of the two channels and the characteristics of the PSFs, both in the in-focus and out-of-focus modes. The in-focus mode yields an FWHM with is smaller than 1px, but a slight (1mm) displacement of the L3 lens on either channel leads to a FWHM of order 2 to 3 pixels and $99 \%$ of the energy in a circle of diameter of about 10 to 12 pixels. 


\begin{tabular}{ll|l|l}
\hline \hline & & Blue channel & Red channel \\
\hline $\begin{array}{l}\text { Field of view } \\
\text { Pixel size }\end{array}$ & & $0.58^{\circ} \times 0.58^{\circ}$ & $0.72^{\circ} \times 0.72^{\circ}$ \\
Wavelength range & & $11 \mu \mathrm{m}=1.06 \mathrm{arcsec}$ & $13 \mu \mathrm{m}=1.3 \mathrm{arcsec}$ \\
\hline PSF diameter & $E=50 \%$ & $400-690 \mathrm{~nm}$ & $690-1000 \mathrm{~nm}$ \\
(in-focus) & $E=90 \%$ & $1.8 \mathrm{px}$ & $0.60-0.70 \mathrm{px}$ \\
& $E=95 \%$ & $3.5 \mathrm{px}$ & $2.3-2.5 \mathrm{px}$ \\
& $E=99 \%$ & $8.9 \mathrm{px}$ & $4.6 \mathrm{px}$ \\
\hline PSF diameter & $E=50 \%$ & $3.06-3.19 \mathrm{px}$ & $11.5 \mathrm{px}$ \\
(out-of-focus) & $E=90 \%$ & $4.61-4.86 \mathrm{px}$ & $4.30-2.98 \mathrm{px}$ \\
& $E=95 \%$ & $5.83-5.93 \mathrm{px}$ & $5.79-6.28 \mathrm{px}$ \\
& $E=99 \%$ & $9.74-9.94 \mathrm{px}$ & $11.6-11.9 \mathrm{px}$ \\
\hline
\end{tabular}

Table 1. Characteristics of the optical solution.

\section{MECHANICAL DESIGN AND THERMALIZATION}

\subsection{Mechanical design of focal box}

The mechanical design was studied by Sophia Engineering (https://www.sophiaengineering.com/en/), based on the scientific and optical specifications. The opto-mechanical modules are fixed on the upper part of the carbon composite plate which is made with a $20 \mathrm{~mm}$ thickness honeycomb sandwich panel. The cameras electronics (power supplies, USB-RJ45 converters, etc.) and the thermalization interfaces are gathered on the other side of carbon plate.

These two parts, opto-mechanical compartment and electronics compartment Figure 5 are separately covered and thermally insulated, the necessary heat for thermalization passes through the two planned holes on the carbon plates.

The focal box upper space (opto-mechanical compartment) is divided in three zones, one for the optical modules and one zone for each camera and thus, each space can be locally thermalized. As for the manufactured material, the design imposes using Titanium alloy for all optical mounts due to its low coefficient of thermal expansion (CTE), excepted the two cameras supports which can be made of aluminium since these modules are located in positive temperature space.
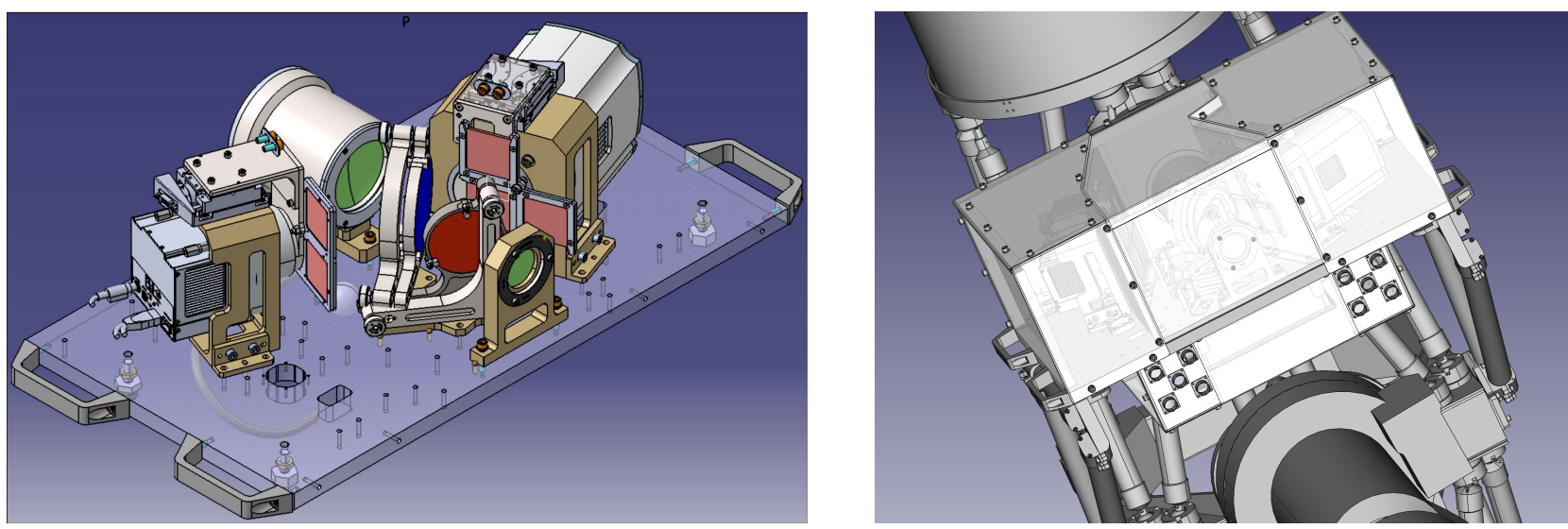

Figure 5. Focal box design: on the left the main carbon composite plat with all opto-mechanic modules, on the right the two closed part of the final box attached to the telescope by fiber carbon structure bars.

On the optical path Figure 6 on the left, at the input of focal box, we have the first module composed of a window and the two first lenses L1/L2 of Wynne field corrector, after that, a dichroic mirror which reflects the blue part of the signal to the FLI camera and a mirror which reflects the red part of the signal to the Andor 
camera. The mounts of these two optical components are put on the same base which allows them to be removed and repositioned during the alignment phases on the telescope. On each optical channel (blue and red), there is a manually rotated filter holder allowing the selection of a specific filter adapted to the type of observation.
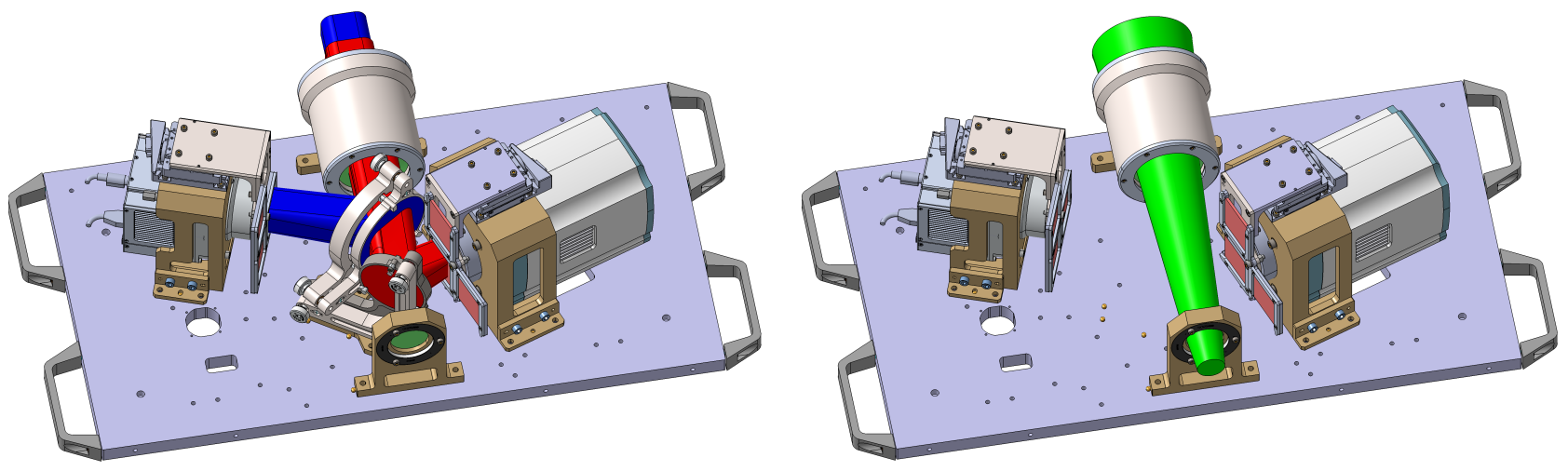

Figure 6. View of the new ASTEP400 focal box optical path and associated opto-mechanical modules.

Finally, the last Wynne field corrector focusing lenses, L3_FLI and L3_Andor, are positioned on mobile modules operated by a PI-MTS65 translation stage which is fixed on each camera support. The support of alignment tools equipped Figure 6 on the right with focusing L3_Alignment lens is planned for the optical telescope M1 and M2 alignment.

\subsection{Thermalisation of the focal box}

The thermalization of focal box is designed to minimize temperature fluctuations and turbulence on the optical path for extreme cases of hot temperature during summer $\left(-30^{\circ} \mathrm{C}\right)$ and extreme cases of cold during winter $\left(-75^{\circ} \mathrm{C}\right)$. For this, we have two compartments one for opto-mechanical modules isolated by Styrodur $20 \mathrm{~mm}$ thick separations with thermal resistance $\mathrm{R}=0.6 \mathrm{~K} \mathrm{~m}^{2} / \mathrm{W}$ and one for the electronics compartment isolated by $6 \mathrm{~mm}$ thick Depron, $\mathrm{R}=0.16 \mathrm{~K} \mathrm{~m}^{2} / \mathrm{W}$. This combination allowing limited heat dissipation at most $130 \mathrm{~W}$ for an outside temperature of $-50^{\circ} \mathrm{C}$. We therefore sized the thermalization heat taking into account, on one hand, this loss and, on the other hand, the heat produced by the electronics of the two sciences cameras. The set point thermalization temperature is around $+10^{\circ} \mathrm{C}$ in camera areas, which allows electronic equipments to operate correctly and $-20^{\circ} \mathrm{C}$ in optical zones.

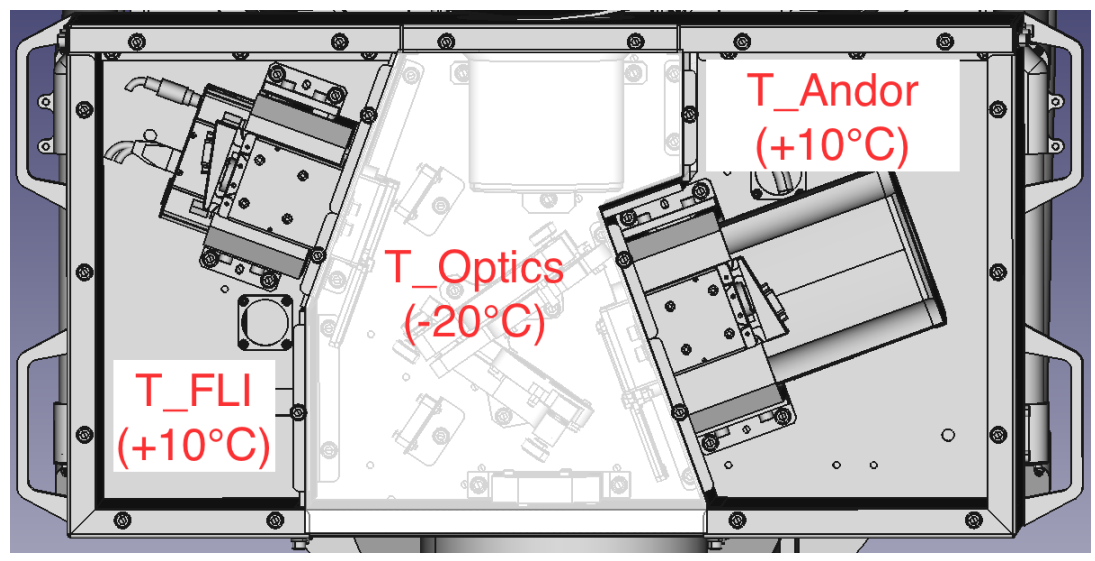

Figure 7. Thermalization zones.

Heating resistances and PT100 temperature sensors, are positioned on each module (iKon camera, FLI, displacement stage, control electronics) and activated by PID (Proportional-Integral-Derivative) regulators to 
maintain the set-point temperature for each component. Fans provide permanent air circulation between the electronic compartment and the two cameras areas. The various temperature set-points will be determined during the test phases in the thermal chamber $\left(-75^{\circ} \mathrm{C}\right)$.

\subsection{Structure modeling and thermal dilatation}

The optical and mechanical design of ASTEP 400 is described elsewhere. ${ }^{8}$ The telescope is a $40 \mathrm{~cm}$ diameter Newtonian type with a carbon tubes for the Serrurier structure. The mount is a commercial AP3600 from Astro-Physics modified to operate in extreme conditions $\left(-75^{\circ} \mathrm{C}\right)$ with a load capacity of $134 \mathrm{~kg}$. In this design the focal box is attached to the Serrurier structure (Figure 8).
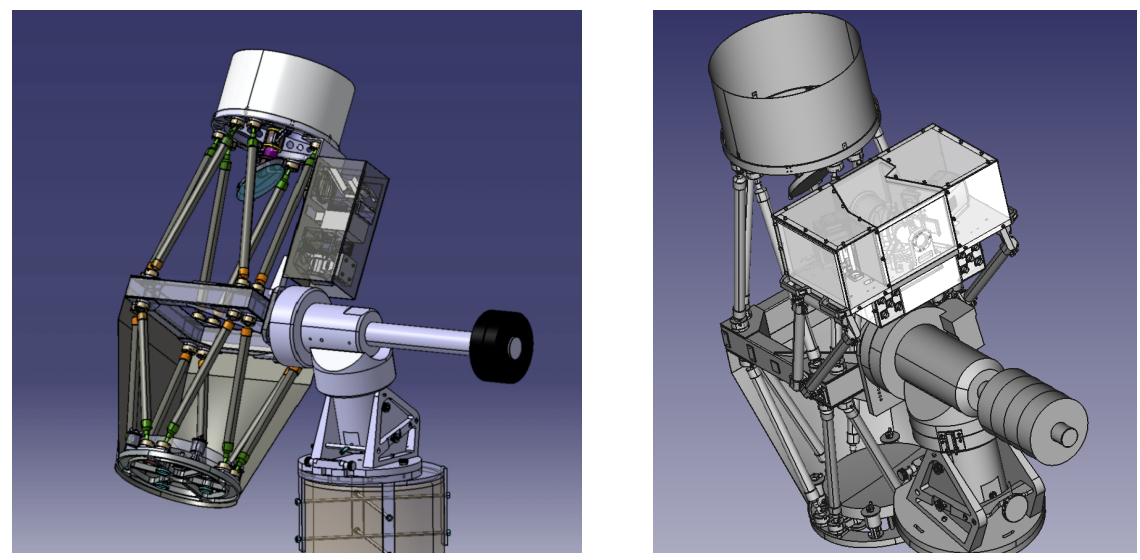

Figure 8. View of the ASTEP 400 telescope design. The previous design is shown on the left, the new design is shown on the right-hand side.

The thermomechanical study and the finite elements modelling of the ASTEP400 telescope structure yields small differential displacements and tilts of the centre of primary mirror (M1), secondary mirror (M2) and the focal box entrance relative to one of four pointing configurations of the telescope over 24 hours observations. This study was based on thermal fluctuations of $30^{\circ} \mathrm{C}$ and a total telescope weight of $83 \mathrm{~kg}$ including $23 \mathrm{~kg}$ for the focal box.

The mechanical design of the new optical configuration required an optimization of geometric configuration of the box and its supporting structure due to the importance of the two sensors weight, $5.9 \mathrm{~kg}$ (iKon-L-936 and FLI KL400) compared to the old ones $3 \mathrm{~kg}$ (FLI Proline and SBIG ME).

For this, a thermo-elastic study of new focal box supporting structure was carried-out in order to meet the ASTEP 400 telescope specifications, new optical design and load limit of the mount. The new focal box is attached to the main part of the Serrurier and fixed on the mount to avoid elasticity stress of the Serrurier structure bar.

The new mechanic study and the modelization by finite elements of the focal box supporting structures have been carried out to minimize the deformations in order to meet the tolerance of stability of camera box position at the level of the entry window maximum $\pm 0.50 \mathrm{~mm}$ in translation and \pm 3 ' in Tip/Tilt.

Models the total weight the structure and the the opto-mechanical elements, cameras, electronics (cables, connectors, etc.) and the cover hoods for the new focal box give an estimate of around $31 \mathrm{~kg}$. The thermal dilatation of the structure was analysed for a $40^{\circ} \mathrm{C}$ thermal gradient. The results obtained by the model of the structure for the four observation orientations of the telescope over 24 hours show a very good stability. In fact, the translation on the three axes $(\mathrm{X}, \mathrm{Y}, \mathrm{Z})$ of the center of the entrance window by the effect of gravity and thermal gradient Figure 8 and Figure 9 is at most $0.24 \mathrm{~mm}$, i.e. $30 \%$ below the specifications. The structure is more efficient in Tip/Tilt with an angle of 1.3 ' at most, i.e. $50 \%$ below the specified limit. 

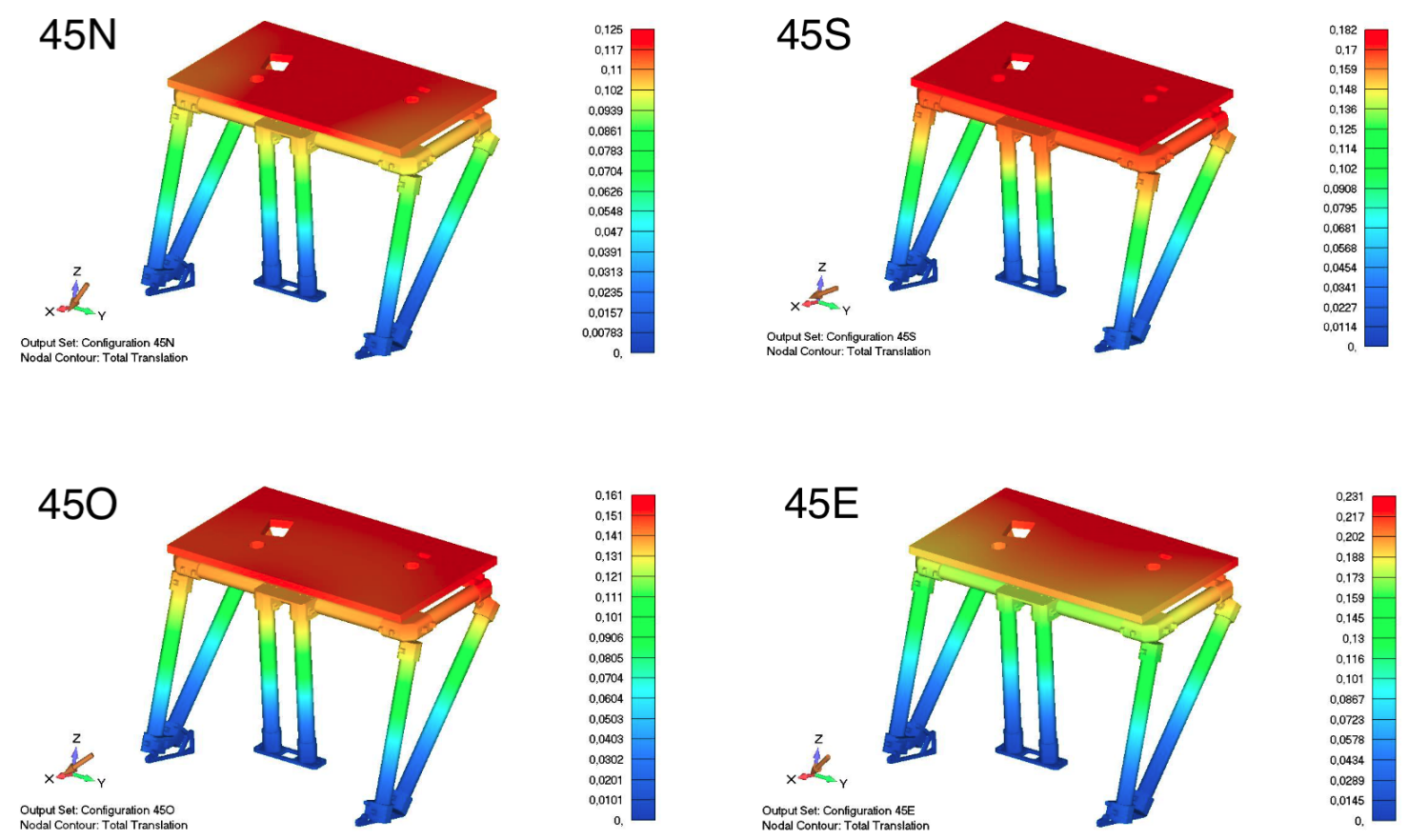

Figure 9. Mechanical deformation of the focal box attach to the telescope structure under the gravity effect according to four telescope orientations cases: Declination of 45 towards the North, Est, South and West

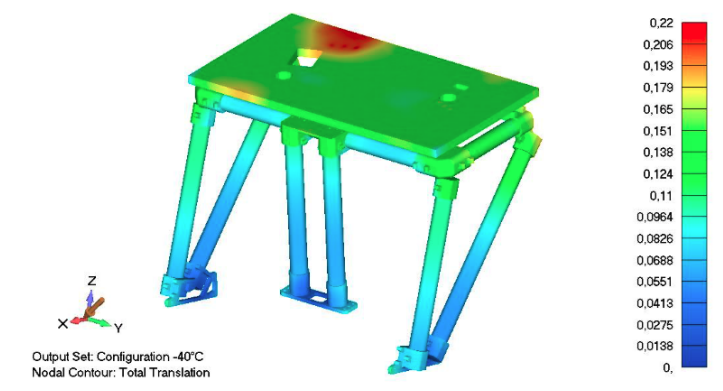

Figure 10. Mechanical structure deformation under the effect of the thermal dilation for $40^{\circ} \mathrm{C}$ gradient.

\section{MANUFACTURING, ASSEMBLY, AND OPERATIONS}

The internal parts for the assembly were manufactured in the mechanical workshop of the Science Payload Validation Section SCI-FIV at ESTEC. The parts were manufactured out of Ti grade 2, using a CNC (Computer Numerical Control) milling machine with carbide cutting tools and cutting fluid. The CAD (Computer-Aided Design) models were used to generate tool paths in a CAM (Computer-Aided Manufacturing) software. The CAM software was then used to generate machine code for the machine to execute the cutting sequences (Figures 11 and 12). Figure 13 shows the camera box on the optical bench at the Fizeau laboratory. The two cameras and lens holders are installed on the carbon fiber plate. Further developments and tests of the optical system are on their way.

The new ASTEP Camera box was to be installed at Concordia at the end of 2020. Because of the COVID-19 pandemic and the subsequent reduction of the scientific operations at the base, installation has been postponed to the end of 2021, with a start of scientific operations on March 2022. The telescope will be operated automatically: 

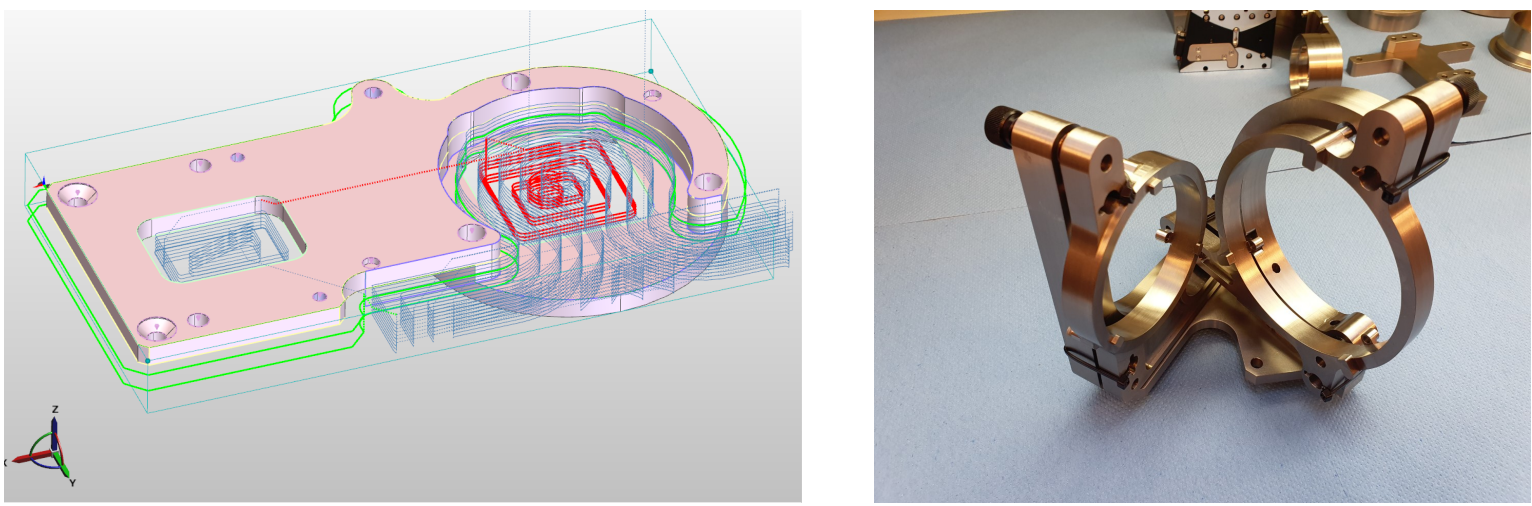

Figure 11. View of part in CAM software with milling features and tool paths (left) and check of the assembly of the dichroic block (right).

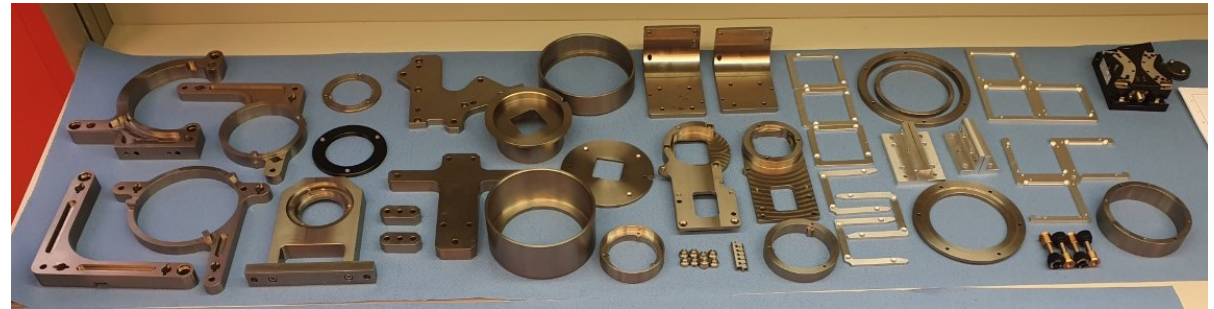

Figure 12. Internal parts of the camera box manufactured at the mechanical workshop of the Science Payload Validation Section SCI-FIV at ESTEC.

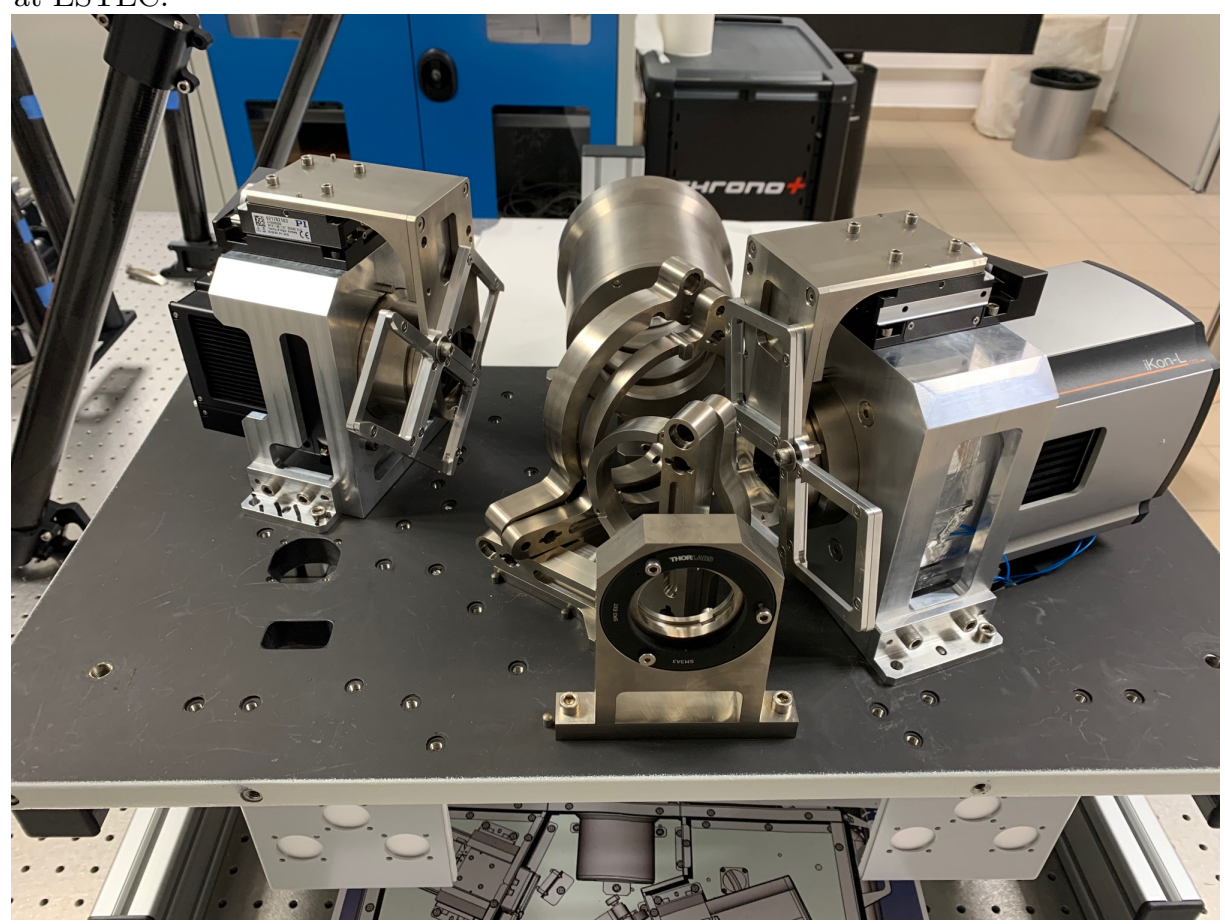

Figure 13. ASTEP+ camera box on the optical bench at the Fizeau laboratory, Université Côte d'Azur. The set includes the FLI camera (left-hand side) with its filter wheel, the set of elements to hold the lenses and mirrors (center) and the Andor camera (right-hand side) with its filter wheel. 
Targets will be chosen by the ASTEP Scientific Committee and uploaded regularly. Once uploaded on the telescope control computer in Concordia, the pointing and guiding is automatic. Alerts are issued to the Nice telescope control team if the star field is not recognized (usually due to bad weather). Due to the limitation of the internet bandwidth, the observation images can be downloaded only in case of need. However, the ASTEP computer server in Concordia is processing the images automatically using an aperture photometry pipeline ${ }^{11}$ and sending control data and lightcurves to the ASTEP team.

\section{EXPECTED PRECISION}

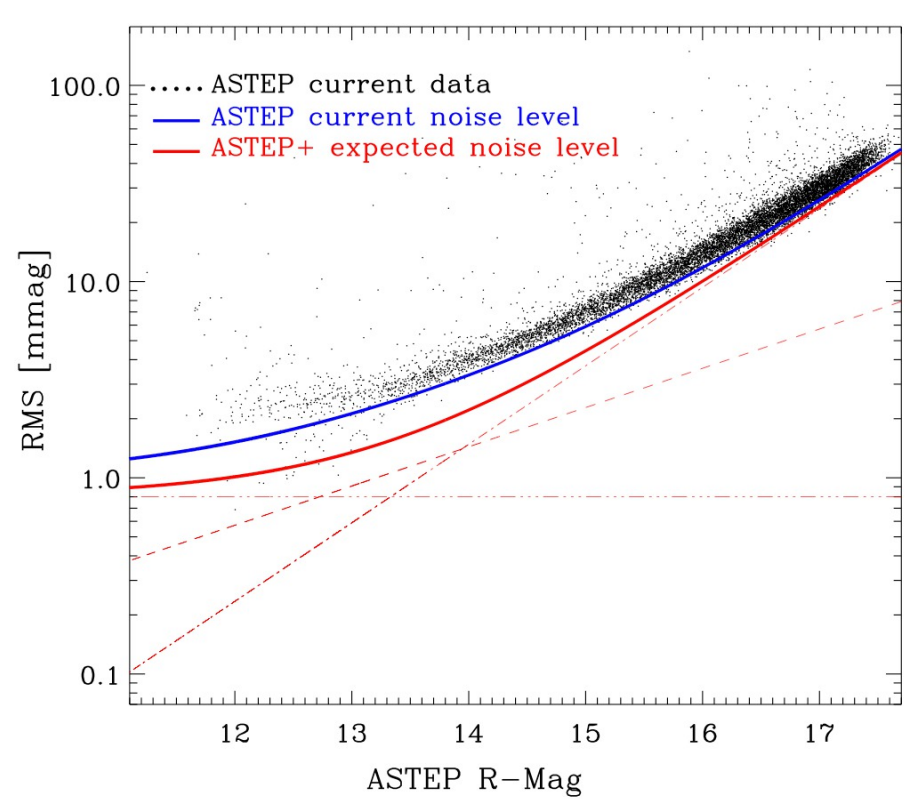

Figure 14. Typical photometric precision attained with the current ASTEP. The group of symbols (black) shows the point to point RMS of light curves for each star, of a typical field, containing more than 10000 stars, and the theoretical photometric precision of the current instrument (blue line). The red dashed, dot-dashed, triple dot dashed and solid line represent the expected source photon noise, sky photon noise, a $800 \mathrm{ppm}$ constant added to account for systematics, and the final precision (quadrature sum of the other three lines) respectively for the new system.

The current photometric precision of ASTEP is $\sim 2$ mmag for bright ( $\mathrm{R} \sim 12 \mathrm{mag})$ stars as described in Ref. 11. With the new system, we expect a photometric gain of about 3.4, which corresponds to an improvement, by a factor of 1.85, of the source photon noise. An improvement of 2.6 can even be achieved by using the correlation between the red and blue channels.

However, effects of correlated noise are significant, particularly for bright stars, and are key limitations of the current system. We identified some guidance to minimize these effects and the new focal box provides some solutions for dealing with these effects. We expect to reach at least a value of $\sim 800 \mathrm{ppm}$ for these systematics.

Fig. 14 displays a typical light curve RMS vs. ASTEP R-band magnitude together together with line representing the theoretical total noise for the current situation. Fig. 14 also displays the expected photon noise, the background noise, a $800 \mathrm{ppm}$ systematic noise and the total noise budget limits expected with the new system. The reduction of the noise level is significant. This result can be further improved if we can decrease the effects of the correlated noise, for example by combining the observations in the two channels. 


\section{CONCLUSION}

We have described the characteristics of the new ASTEP+ camera box. When installed on the telescope based at Concordia, Antarctica, at the end of 2021, it will allow accurate photometric observations of stars with exoplanets simultaneously in the blue and red channels. This new capability will enable collecting and effectively using for science purposes three times more photons than the previous camera. Thanks to the two-color observations, it will also enable a more efficient determination of chromatic effects, important to separate bona-fide transiting planets from background eclipsing binaries.

With the forthcoming launch of JWST and, a bit further down the road, of Ariel, the possibility to have an observatory in Antarctica is crucial, in particular to monitor and prepare the observations of these missions by a first characterization of the objects to be observed and a refinement of their ephemerides, in the case of transiting exoplanets. Indeed, Concordia is ideally located to observe the southern continuous viewing zones of these two telescopes which corresponds to a circumpolar region, i.e. a region of the sky which never sets, allowing a continuous viewing by ASTEP during the southern winter. This was exemplified by the successful monitoring of $\beta$-Pictoris ${ }^{13,24}$ in 2017 and 2018 and the follow-up of TESS targets starting in 2019. ${ }^{25}$ The exploration of exoplanets in our Galaxy will require many observing facilities for the extremely important and massive follow-up work that is needed. With its new two-wavelength approach, ASTEP+ will provide much needed photometric observations of exoplanets and exoplanetary candidates.

\section{ACKNOWLEDGMENTS}

The ASTEP project was funded by the Agence Nationale de la Recherche (ANR), the Institut National des Sciences de l'Univers (INSU), the Programme National de Planétologie (PNP), the Observatoire de la Côte d'Azur and the Université Côte d'Azur through Idex UCA JEDI (ANR-15-IDEX-01). The logistics at Concordia is handled by the French Institut Paul-Emile Victor (IPEV) and the Italian Programma Nazionale di Ricerche in Antartide (PNRA). This research received funding from the European Research Council (ERC) under the European Union's Horizon 2020 research and innovation programme (grant agreement $\mathrm{n}^{\circ}$ 803193/BEBOP), and from the Science and Technology Facilities Council (STFC; grant $n^{\circ}$ ST/S00193X/1). This work made use of the NASA's Astrophysics Data System bibliographic services.

\section{REFERENCES}

[1] Kenyon, S. L. and Storey, J. W. V., "A Review of Optical Sky Brightness and Extinction at Dome C, Antarctica," PASP 118, 489-502 (Mar. 2006).

[2] Lawrence, J. S., "Infrared and Submillimeter Atmospheric Characteristics of High Antarctic Plateau Sites," PASP 116, 482-492 (May 2004).

[3] Crouzet, N., Chapellier, E., Guillot, T., Mékarnia, D., Agabi, A., Fanteï-Caujolle, Y., Abe, L., Rivet, J.-P., Schmider, F.-X., Fressin, F., Bondoux, E., Challita, Z., Pouzenc, C., Valbousquet, F., Bayliss, D., Bonhomme, S., Daban, J.-B., Gouvret, C., and Blazit, A., "Four winters of photometry with ASTEP South at Dome C, Antarctica," A\&A 619, A116 (Nov. 2018).

[4] Crouzet, N., Guillot, T., Agabi, A., Rivet, J.-P., Bondoux, E., Challita, Z., Fanteï-Caujolle, Y., Fressin, F., Mékarnia, D., Schmider, F.-X., Valbousquet, F., Blazit, A., Bonhomme, S., Abe, L., Daban, J.-B., Gouvret, C., Fruth, T., Rauer, H., Erikson, A., Barbieri, M., Aigrain, S., and Pont, F., "ASTEP South: an Antarctic Search for Transiting ExoPlanets around the celestial south pole," A\&A 511, A36 (Feb. 2010).

[5] Aristidi, E., Agabi, K., Azouit, M., Fossat, E., Vernin, J., Travouillon, T., Lawrence, J. S., Meyer, C., Storey, J. W. V., Halter, B., Roth, W. L., and Walden, V., "An analysis of temperatures and wind speeds above Dome C, Antarctica," A\&A 430, 739-746 (Feb. 2005).

[6] Kenyon, S. L., Lawrence, J. S., Ashley, M. C. B., Storey, J. W. V., Tokovinin, A., and Fossat, E., "Atmospheric Scintillation at Dome C, Antarctica: Implications for Photometry and Astrometry," PASP 118, 924-932 (June 2006).

[7] Fressin, F., Guillot, T., Bouchy, F., Erikson, A., Gay, J., Léger, A., Pont, F., Rauer, H., Rivet, J., and Valbousquet, F., "Antarctica Search for Transiting Extrasolar Planets," in [EAS Publications Series], M. Giard, F. Casoli, \& F. Paletou, ed., EAS Publications Series 14, 309-312 (2005). 
[8] Daban, J.-B., Gouvret, C., Guillot, T., Agabi, A., Crouzet, N., Rivet, J.-P., Mekarnia, D., Abe, L., Bondoux, E., Fanteï-Caujolle, Y., Fressin, F., Schmider, F.-X., Valbousquet, F., Blanc, P.-E., Le van Suu, A., Rauer, H., Erikson, A., Pont, F., and Aigrain, S., "ASTEP 400: a telescope designed for exoplanet transit detection from Dome C, Antarctica," in [Ground-based and Airborne Telescopes III], Proc. SPIE 7733, 77334T (July 2010).

[9] Abe, L., Gonçalves, I., Agabi, A., Alapini, A., Guillot, T., Mékarnia, D., Rivet, J.-P., Schmider, F.-X., Crouzet, N., Fortney, J., Pont, F., Barbieri, M., Daban, J.-B., Fanteï-Caujolle, Y., Gouvret, C., Bresson, Y., Roussel, A., Bonhomme, S., Robini, A., Dugué, M., Bondoux, E., Péron, S., Petit, P.-Y., Szulágyi, J., Fruth, T., Erikson, A., Rauer, H., Fressin, F., Valbousquet, F., Blanc, P.-E., Le van Suu, A., and Aigrain, S., "The secondary eclipses of WASP-19b as seen by the ASTEP 400 telescope from Antarctica," A\&A 553, A49 (May 2013).

[10] Guillot, T., Abe, L., Agabi, A., Rivet, J. P., Daban, J. B., Mékarnia, D., Aristidi, E., Schmider, F. X., Crouzet, N., Gonçalves, I., Gouvret, C., Ottogalli, S., Faradji, H., Blanc, P. E., Bondoux, E., and Valbousquet, F., "Thermalizing a telescope in Antarctica - analysis of ASTEP observations," Astronomische Nachrichten 336, 638 (Sept. 2015).

[11] Mékarnia, D., Guillot, T., Rivet, J.-P., Schmider, F.-X., Abe, L., Gonçalves, I., Agabi, A., Crouzet, N., Fruth, T., Barbieri, M., Bayliss, D. D. R., Zhou, G., Aristidi, E., Szulagyi, J., Daban, J.-B., Fanteï-Caujolle, Y., Gouvret, C., Erikson, A., Rauer, H., Bouchy, F., Gerakis, J., and Bouchez, G., "Transiting planet candidates with ASTEP 400 at Dome C, Antarctica," MNRAS 463, 45-62 (Nov. 2016).

[12] Chapellier, E., Mékarnia, D., Abe, L., Guillot, T., Agabi, K., Rivet, J.-P., Schmider, F.-X., Crouzet, N., and Aristidi, E., "A Catalog of Eclipsing Binaries and Variable Stars Observed with ASTEP 400 from Dome C, Antarctica," ApJS 226, 21 (Oct. 2016).

[13] Mékarnia, D., Chapellier, E., Guillot, T., Abe, L., Agabi, A., De Pra, Y., Schmider, F.-X., Zwintz, K., Stevenson, K. B., Wang, J. J., Lagrange, A.-M., Bigot, L., Crouzet, N., Fanteï-Caujolle, Y., Christille, J.M., and Kalas, P., "The $\delta$ Scuti pulsations of $\beta$ Pictoris as observed by ASTEP from Antarctica," A\&A 608, L6 (Dec. 2017).

[14] Dressing, C. D. and Charbonneau, D., "The Occurrence Rate of Small Planets around Small Stars," ApJ 767, 95 (Apr. 2013).

[15] Morton, T. D. and Swift, J., "The Radius Distribution of Planets around Cool Stars," ApJ 791, 10 (Aug. 2014).

[16] Dressing, C. D. and Charbonneau, D., "The Occurrence of Potentially Habitable Planets Orbiting M Dwarfs Estimated from the Full Kepler Dataset and an Empirical Measurement of the Detection Sensitivity," ApJ 807, 45 (July 2015).

[17] Doyle, L. R., Carter, J. A., Fabrycky, D. C., Slawson, R. W., Howell, S. B., Winn, J. N., Orosz, J. A., Přsa, A., Welsh, W. F., Quinn, S. N., Latham, D., Torres, G., Buchhave, L. A., Marcy, G. W., Fortney, J. J., Shporer, A., Ford, E. B., Lissauer, J. J., Ragozzine, D., Rucker, M., Batalha, N., Jenkins, J. M., Borucki, W. J., Koch, D., Middour, C. K., Hall, J. R., McCauliff, S., Fanelli, M. N., Quintana, E. V., Holman, M. J., Caldwell, D. A., Still, M., Stefanik, R. P., Brown, W. R., Esquerdo, G. A., Tang, S., Furesz, G., Geary, J. C., Berlind, P., Calkins, M. L., Short, D. R., Steffen, J. H., Sasselov, D., Dunham, E. W., Cochran, W. D., Boss, A., Haas, M. R., Buzasi, D., and Fischer, D., "Kepler-16: A Transiting Circumbinary Planet," Science 333, 1602 (Sept. 2011).

[18] Martin, D. V., [Populations of Planets in Multiple Star Systems], 156 (2018).

[19] Ricker, G. R., Winn, J. N., Vanderspek, R., Latham, D. W., Bakos, G. Á., Bean, J. L., Berta-Thompson, Z. K., Brown, T. M., Buchhave, L., Butler, N. R., Butler, R. P., Chaplin, W. J., Charbonneau, D., Christensen-Dalsgaard, J., Clampin, M., Deming, D., Doty, J., De Lee, N., Dressing, C., Dunham, E. W., Endl, M., Fressin, F., Ge, J., Henning, T., Holman, M. J., Howard, A. W., Ida, S., Jenkins, J., Jernigan, G., Johnson, J. A., Kaltenegger, L., Kawai, N., Kjeldsen, H., Laughlin, G., Levine, A. M., Lin, D., Lissauer, J. J., MacQueen, P., Marcy, G., McCullough, P. R., Morton, T. D., Narita, N., Paegert, M., Palle, E., Pepe, F., Pepper, J., Quirrenbach, A., Rinehart, S. A., Sasselov, D., Sato, B., Seager, S., Sozzetti, A., Stassun, K. G., Sullivan, P., Szentgyorgyi, A., Torres, G., Udry, S., and Villasenor, J., "Transiting Exoplanet Survey Satellite (TESS)," in [Society of Photo-Optical Instrumentation Engineers (SPIE) Conference Series], Society of Photo-Optical Instrumentation Engineers (SPIE) Conference Series 9143, 20 (Aug. 2014). 
[20] Wheatley, P. J., West, R. G., Goad, M. R., Jenkins, J. S., Pollacco, D. L., Queloz, D., Rauer, H., Udry, S., Watson, C. A., Chazelas, B., Eigmüller, P., Lambert, G., Genolet, L., McCormac, J., Walker, S., Armstrong, D. J., Bayliss, D., Bento, J., Bouchy, F., Burleigh, M. R., Cabrera, J., Casewell, S. L., Chaushev, A., Chote, P., Csizmadia, S., Erikson, A., Faedi, F., Foxell, E., Gänsicke, B. T., Gillen, E., Grange, A., Günther, M. N., Hodgkin, S. T., Jackman, J., Jordán, A., Louden, T., Metrailler, L., Moyano, M., Nielsen, L. D., Osborn, H. P., Poppenhaeger, K., Raddi, R., Raynard, L., Smith, A. M. S., Soto, M., and Titz-Weider, R., "The Next Generation Transit Survey (NGTS)," MNRAS 475, 4476-4493 (Apr. 2018).

[21] Delrez, L., Gillon, M., Queloz, D., Demory, B.-O., Almleaky, Y., de Wit, J., Jehin, E., Triaud, A. H. M. J., Barkaoui, K., Burdanov, A., Burgasser, A. J., Ducrot, E., McCormac, J., Murray, C., Silva Fernandes, C., Sohy, S., Thompson, S. J., Van Grootel, V., Alonso, R., Benkhaldoun, Z., and Rebolo, R., "SPECULOOS: a network of robotic telescopes to hunt for terrestrial planets around the nearest ultracool dwarfs," in [Ground-based and Airborne Telescopes VII], Marshall, H. K. and Spyromilio, J., eds., Society of PhotoOptical Instrumentation Engineers (SPIE) Conference Series 10700, 107001I (July 2018).

[22] Murray, C. A., Delrez, L., Pedersen, P. P., Queloz, D., Gillon, M., Burdanov, A., Ducrot, E., Garcia, L. J., Lienhard, F., Demory, B. O., Jehin, E., McCormac, J., Sebastian, D., Sohy, S., Thompson, S. J., Triaud, A. H. M. J., Van Grootel, V., Günther, M. N., and Huang, C. X., "Photometry and performance of SPECULOOS-South," MNRAS 495, 2446-2457 (May 2020).

[23] Sebastian, D., Gillon, M., Ducrot, E., Pozuelos, F. J., Garcia, L. J., Günther, M. N., Delrez, L., Queloz, D., Demory, B. O., Triaud, A. H. M. J., Burgasser, A., de Wit, J., Burdanov, A., Dransfield, G., Jehin, E., McCormac, J., Murray, C. A., Niraula, P., Pedersen, P. P., Rackham, B. V., Sohy, S., Thompson, S., and Van Grootel, V., "SPECULOOS - Ultracool Dwarf Transit Survey: Target List and Strategy," arXiv e-prints, arXiv:2011.02069 (Nov. 2020).

[24] Lagrange, A. M., Meunier, N., Rubini, P., Keppler, M., Galland, F., Chapellier, E., Michel, E., Balona, L., Beust, H., Guillot, T., Grandjean, A., Borgniet, S., Mékarnia, D., Wilson, P. A., Kiefer, F., Bonnefoy, M., Lillo-Box, J., Pantoja, B., Jones, M., Iglesias, D. P., Rodet, L., Diaz, M., Zapata, A., Abe, L., and Schmider, F.-X., "Evidence for an additional planet in the $\beta$ Pictoris system," Nature Astronomy 3, 1135-1142 (Aug. 2019).

[25] Bouma, L. G., Hartman, J. D., Brahm, R., Evans, P., Collins, K. A., Zhou, G., Sarkis, P., Quinn, S. N., de Leon, J., Livingston, J., Bergmann, C., Stassun, K. G., Bhatti, W., Winn, J. N., Bakos, G. Á., Abe, L., Crouzet, N., Dransfield, G., Guillot, T., Marie-Sainte, W., Mékarnia, D., Triaud, A. H. M. J., Tinney, C. G., Henning, T., Espinoza, N., Jordán, A., Barbieri, M., Nandakumar, S., Trifonov, T., Vines, J. I., Vuckovic, M., Ziegler, C., Law, N., Mann, A. W., Ricker, G. R., Vanderspek, R., Seager, S., Jenkins, J. M., Burke, C. J., Dragomir, D., Levine, A. M., Quintana, E. V., Rodriguez, J. E., Smith, J. C., and Wohler, B., "Cluster Difference Imaging Photometric Survey. II. TOI 837: A Young Validated Planet in IC 2602," AJ 160, 239 (Nov. 2020). 